\title{
Artificial Wooden Nacre: A High Specific Strength Engineering Material
}

Yipeng Chen, ${ }^{1}$ Jinzhou Fu, ${ }^{2}$ Baokang Dang, ${ }^{\text {I }}$ Qingfeng Sun, ${ }^{1, *}$ Huiqiao Li, ${ }^{2,3, *}$ Tianyou Zhai ${ }^{2,3}$

${ }^{1}$ School of Engineering, Zhejiang A\&F University, Hangzhou 311300, China

${ }^{2}$ State Key Laboratory of Material Processing and Die \& Mould Technology, School of Materials Science and Engineering, Huazhong University of Science and Technology, Wuhan 430074, China

${ }^{3}$ Shenzhen Research Institute of Huazhong University of Science and Technology, Shenzhen 518000, China

*Corresponding author: Qingfeng Sun (qfsun@zafu.edu.cn) and Huiqiao Li (hqli@hust.edu.cn) 
S1 Calculation of the fracture toughness $K_{\mathrm{Jc}}$ and the crack extension resistance

curve. The crack extension resistance curve, also known as $R$-curve, is used to evaluate the toughening behavior of the lignocellulosic nacre. The $R$-curves are developed based on SENB tests. The converted stress intensity factor $K_{J c}$ is determined by

$$
K_{J c}=\sqrt{\left(J_{e l}+J_{p l}\right) E^{\prime}}
$$

where $J_{e l}$ is the elastic component of $\mathrm{J}$-integral, $J_{p l}$ the plastic component of $J$-integral

and $E^{\prime}$ is given by

$$
E^{\prime}=\frac{E}{1-v^{2}}
$$

for plane strain conditions, where E is the elastic modulus and $v$ the Poisson's ratio.

The calculation of $J_{e l}$ is based on the relation

$$
J_{e l}=\frac{K_{I c}^{2}}{E^{\prime}},
$$

where $K_{I c}$ is the plane strain fracture toughness at or near to the onset of crack initiation.

$K_{I c}$ is given by

$$
K_{I C}=\frac{P S}{B W^{\frac{3}{2}}} f\left(\frac{a}{W}\right),
$$

where $\mathrm{P}$ is the applied load, $\mathrm{S}$ the span, B the width of the SENB specimen, $W$ the thickness and $a$ the initial crack length. The initial crack length $a$ is equal to the depth of the notch for SENB specimens. The function $f$ is given by

$$
f\left(\frac{a}{W}\right)=\frac{3\left(\frac{a}{w}\right)^{\frac{1}{2}}\left[1.99-\frac{a}{w}\left(1-\frac{a}{w}\right)\left(2.15-3.93 \frac{a}{W}+2.7\left(\frac{a}{w}\right)^{2}\right)\right]}{2\left(1+2 \frac{a}{W}\right)\left(1-\frac{a}{w}\right)^{\frac{3}{2}}} .
$$


The plastic component $J_{p l}$ is calculated by

$$
J_{p l}=\frac{2 A_{p l}}{B b}
$$

where $A_{p l}$ is the area of the plastic region under load-displacement curve and $\mathrm{b}$ the crack remaining ligament. The crack length is calculated using a recursive equation:

$$
a_{n}=a_{n-1}+\frac{W-a_{n-1} C_{n}-C_{n-1}}{2}
$$

where the compliance $C_{n}$ is given by

$$
C=\frac{u}{f}
$$

where $u$ is the displacement and $f$ the load of each point after crack initiation.

\section{S2 Finite element analysis of the brick-and-mortar structure.}

A three-dimensional (3D) nonlinear finite element model is developed using the commercial software ABAQUS v6.13. In the simulation, a 3D brick-and-mortar (BM) structure $\left(250 \times 60 \times 2 \mu \mathrm{m}^{3}\right)$ with a single edge notch $\left(2 \times 4 \times 2 \mu \mathrm{m}^{3}\right)$ is adopted, as shown in Figure S13. The BM structure in the FE model contains a randomly staggered arrangement of bricks bonded by the thin layer of bio-polymer which is modeled as a cohesive zone with a bilinear traction-separation and undergoes dry friction after damage. The bricks with isotropic bulk modulus $E p=20$ GPa, Poisson ratio $v p=0.28$ and the failure strength $\sigma_{p}^{m}=60 \mathrm{MPa}$ bear elastic deformation before brittle failure. 

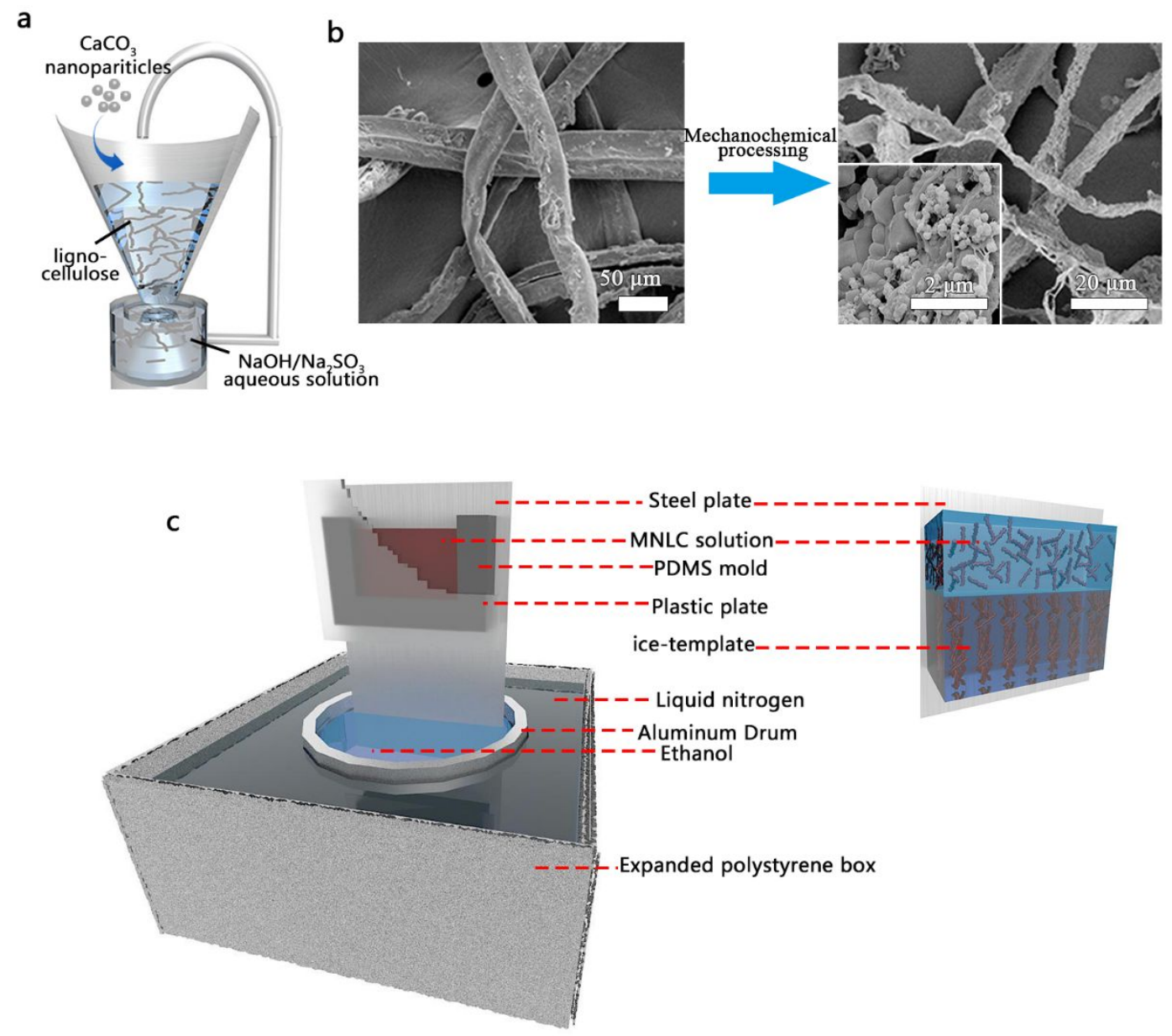

Figure S1. (a) Mechanochemical and delignified process of lignocellulose and $\mathrm{CaCO}_{3}$. During mechanochemical processing, lignocellulose undergoes splitting, breaking, deformation, volumetric changes, and other physical changes. Then, the intrant $\mathrm{CaCO}_{3}$ were adhered to the lignocellulose surface by hydrogen bonding and electrostatic adsorption force. (b) SEM image of lignocellulose (left) and lignocellulosic building block (right). (c) (left) Experimental setup of the freeze-casting process. The temperature of the ethanol is kept constant at $-75^{\circ} \mathrm{C}$, which can be controlled via adding liquid nitrogen. (right) Mechanism of the formation of the laminated DMNLC matrix driven by the crystallization of ice. 

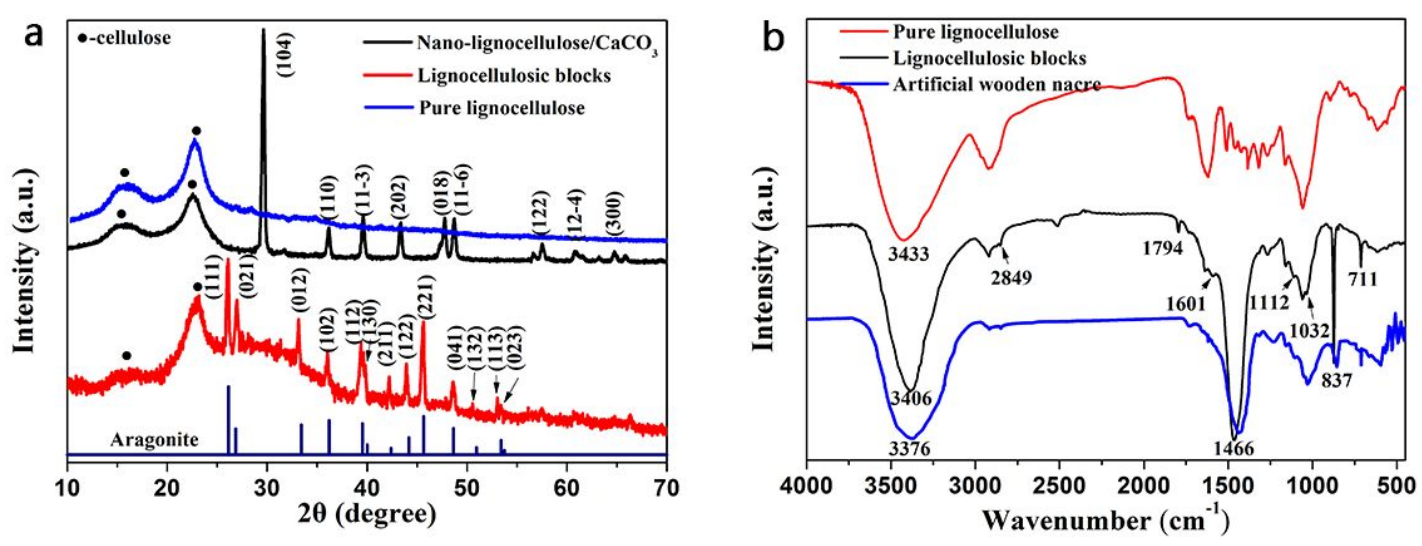

Figure S2. (a) XRD of pure lignocellulose, nano-lignocellulose $/ \mathrm{CaCO}_{3}$ composite, lignocellulosic blocks and pure aragonite. (b) FTIR spectra of pure lignocellulose, lignocellulosic blocks and artificial wooden nacres. The absorption peak of the lignocellulosic blocks at $1466 \mathrm{~cm}^{-1}, 711 \mathrm{~cm}^{-1}$ and $873 \mathrm{~cm}^{-1}$ was due to the existence of $\mathrm{CaCO}_{3}$, which indicate the mineralization of lignocellulose. The shift of maximum absorption hydroxyl peak of artificial wooden nacre from $3406 \mathrm{~cm}^{-1}$ to the lower $3376 \mathrm{~cm}^{-1}$ indicates that hydroxyl group can still form hydrogen bond during the process of hot pressing, which is conducive to the self-gluing of lignocellulosic blocks into layers. 


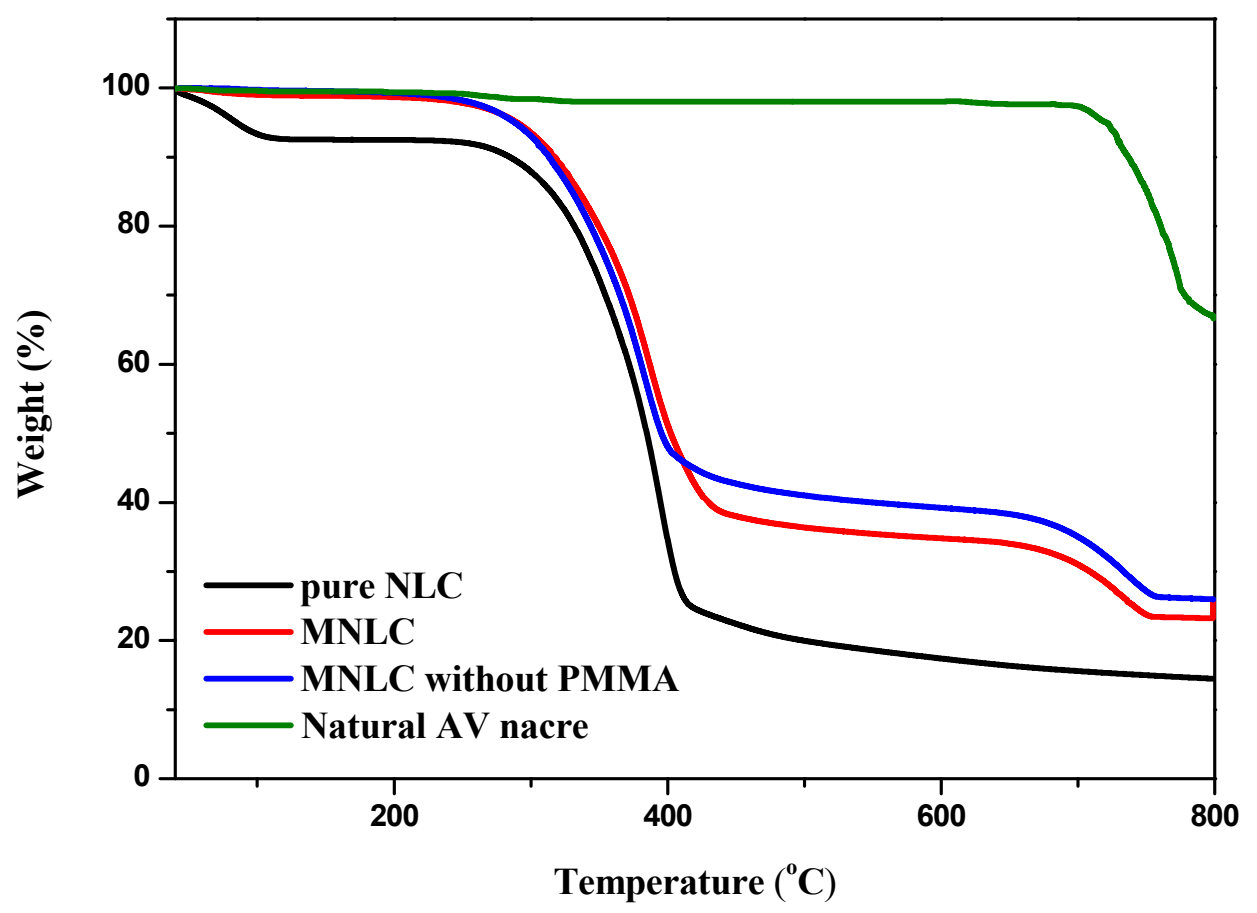

Figure S3. Compositional analysis of artificial nacre. Thermogravimetric analysis (TGA) of lignocellulosic building blocks showing low and high temperature weight loss from dehydration crystallisation and PMMA degradation within the lignocellulosic building blocks, respectively. The content of water, PMMA and $\mathrm{CaCO}_{3}$ is calculated to be $3 \mathrm{wt} \%, 1.3 \mathrm{wt} \%$, and $15.6 \mathrm{wt} \%$, respectively. The content of $\mathrm{CaCO}_{3}$ in natural AV nacre is calculated to be $96.3 \mathrm{wt} \%$. 


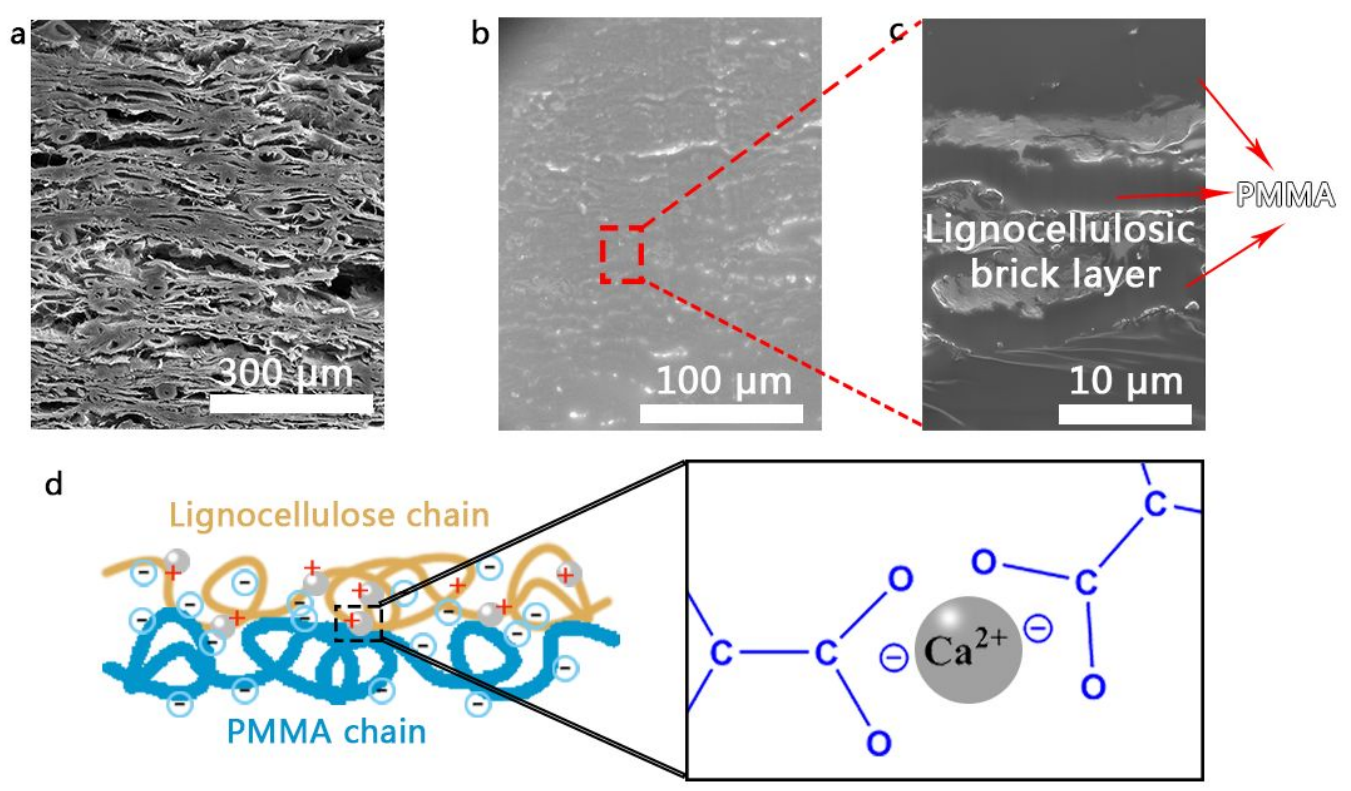

Figure S4. (a) The scanning electron micrograph (SEM) of the cross section of the lignocellulosic brick layers without PMMA. (b) and (c) The SEM of the cross section of PMMA layer between two lignocellulosic brick layers. (d) A structural model for the segregated amorphous composite consisting of lignocellulosic brick and PMMA. 

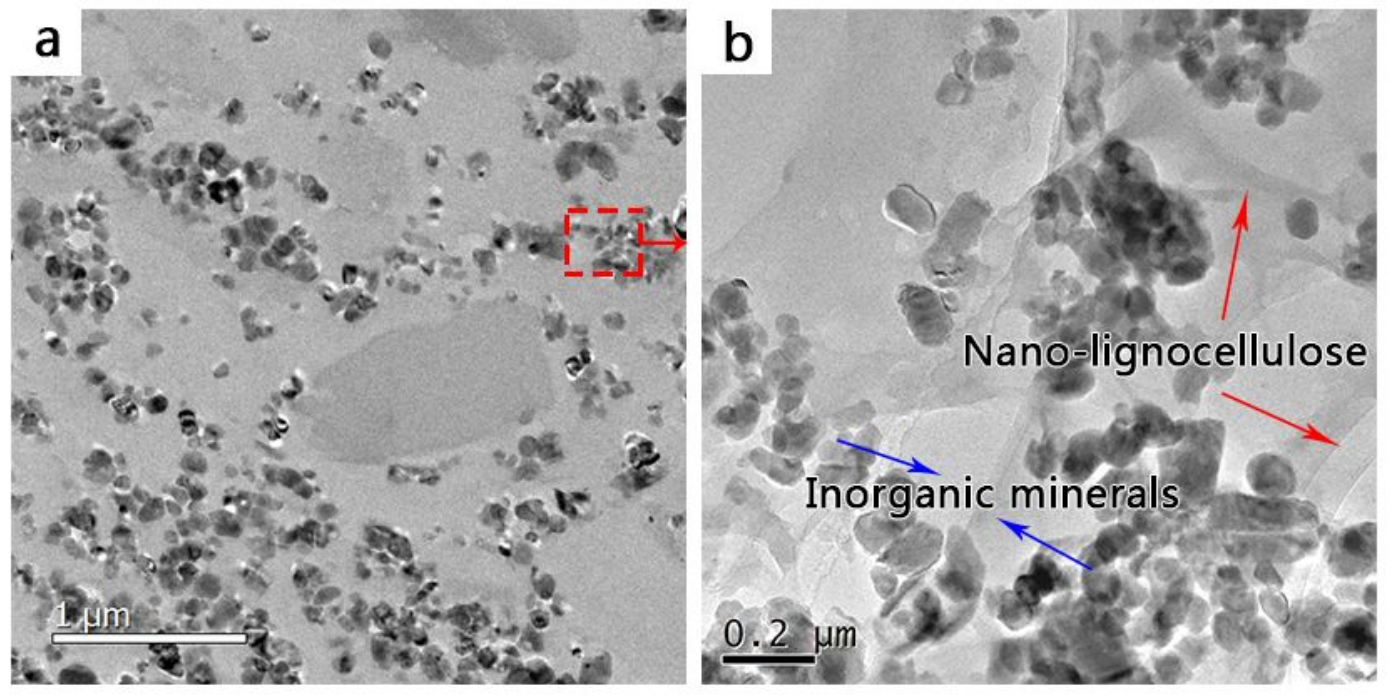

Figure S5. (a) TEM image of the lignocellulosic brick layer of the artificial wooden nacre. (b) HRTEM image of the selected area in (a) 

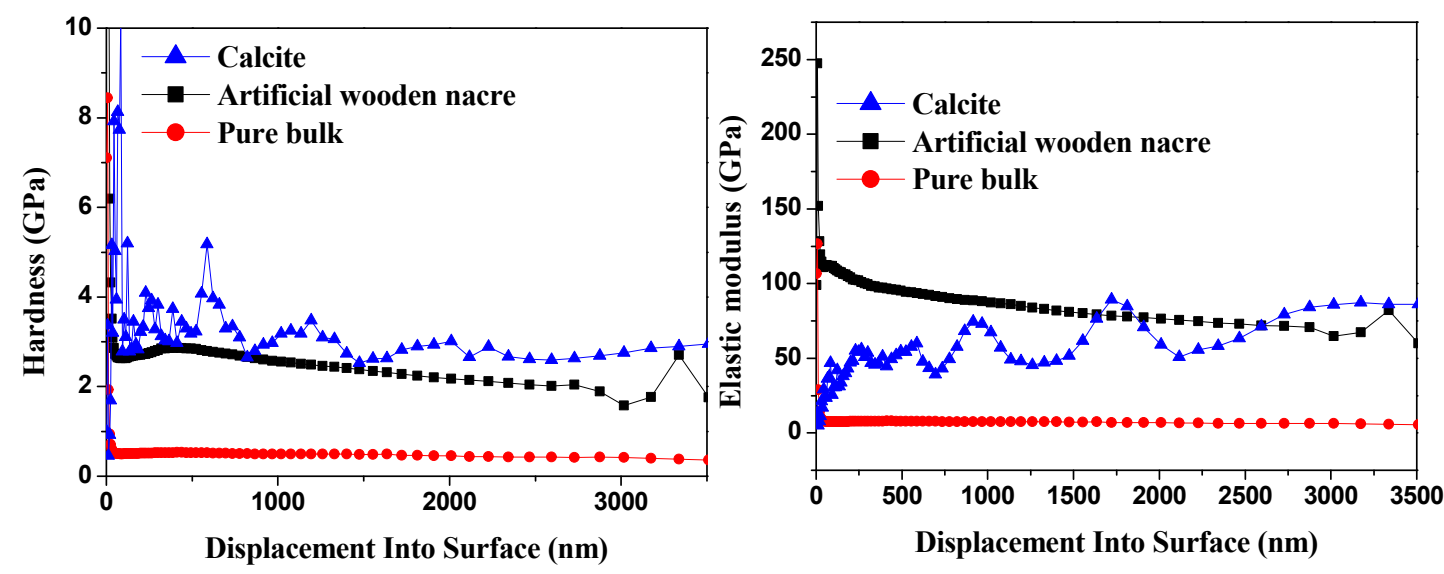

Figure S6. (a) Elastic modulus and (b) hardness curve of calcite, pure delignified nano-lignocellulose and artificial wooden nacre. 

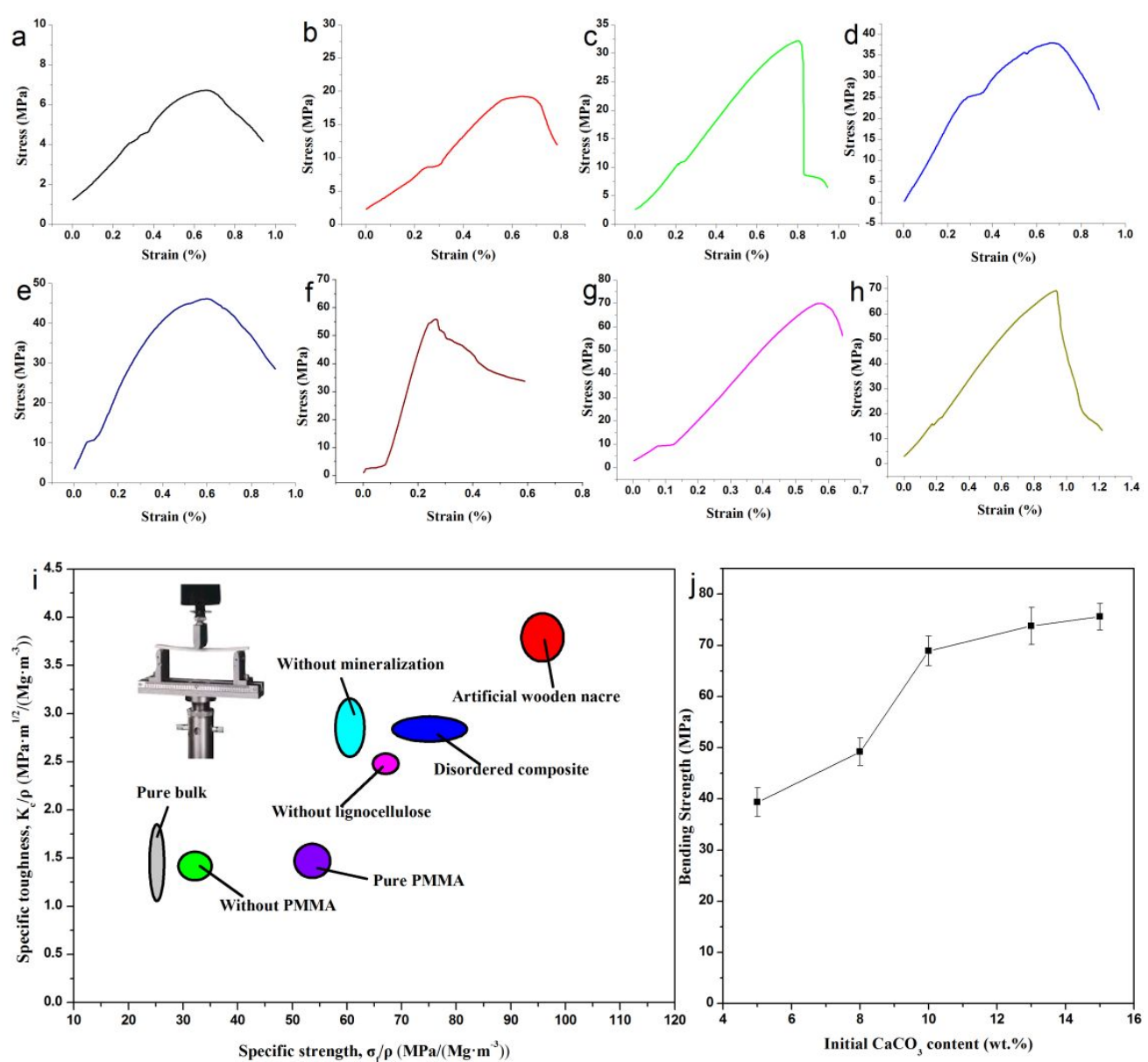

Figure S7. Stress-strain curve of (a) blended mixture, (b) pure delignified nano-lignocellulose, (c) disordered composite, (d) artificial wooden nacre without mineralization, (e) artificial wooden nacre without PMMA, (f) pure PMMA, (g) natural Atrina vexillum nacre and (h) the artificial wooden nacre. (i) Specific fracture toughness versus specific ultimate flexural strength, illustrating the mechanical performance of the artificial wooden nacre and their related materials. (j) Bending strength of the artificial wooden nacre with the different initial concentrations of $\mathrm{CaCO}_{3}$ 
a
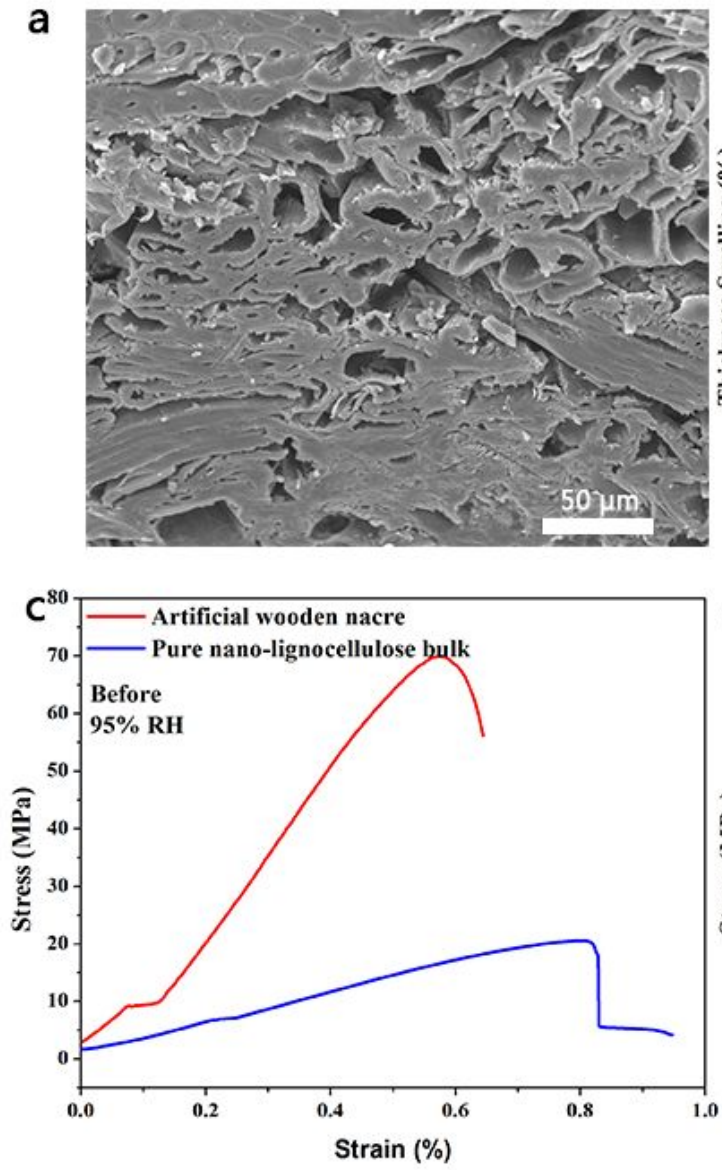
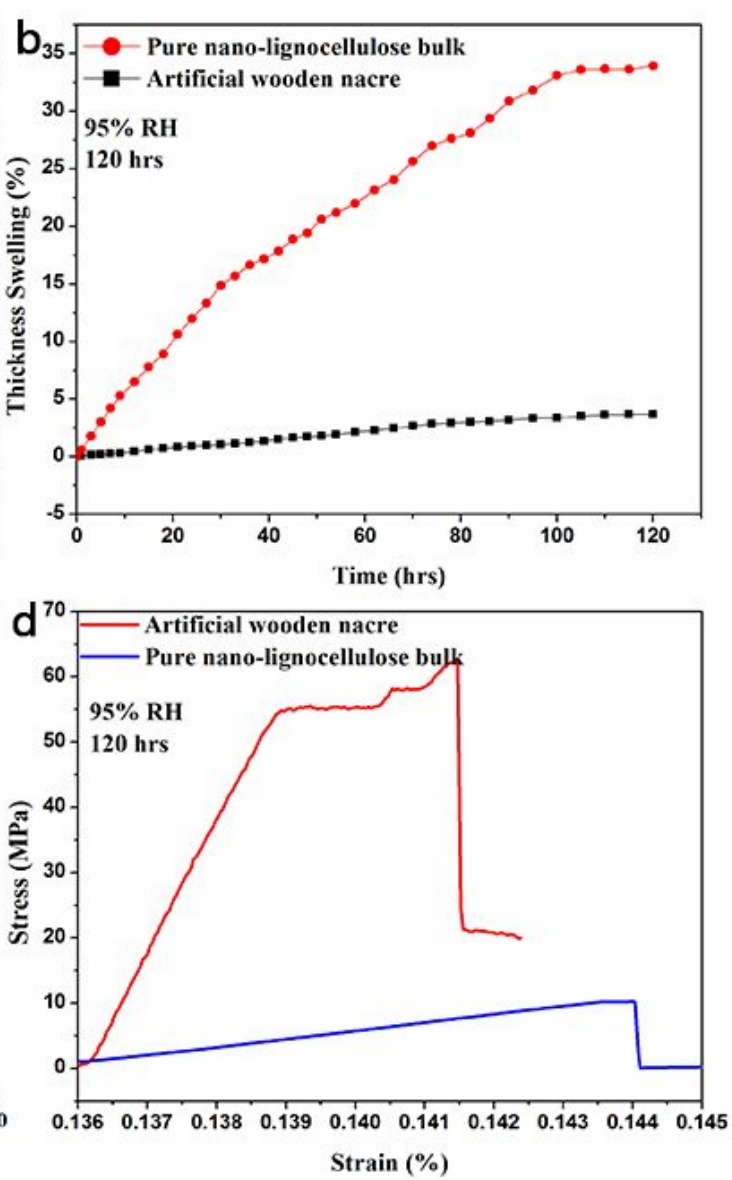

Figure S8. (a) Change in thickness of the artificial wooden nacre and pure delignified nano-lignocellulose bulk over time. (b) Flexural stress-strain curves of the two samples after sustaining 95\% RH for $120 \mathrm{~h}$. 

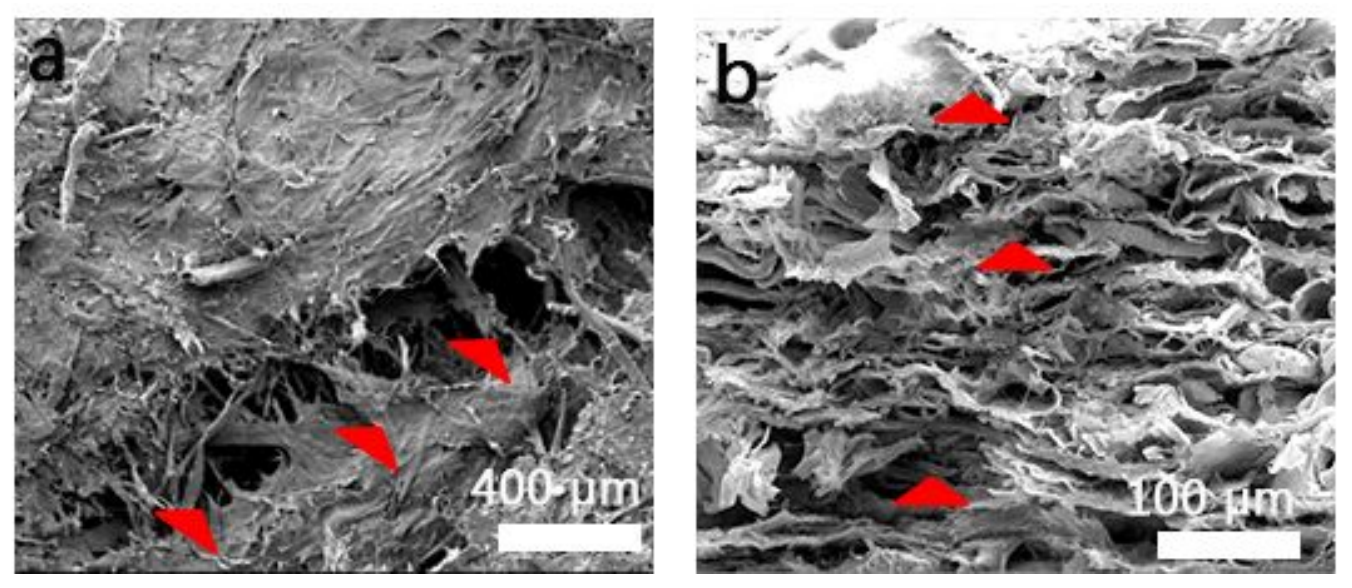

Figure S9. (a) Tension-induced crack of the lignocellulosic nacre reveals the bridging of the lignocellulosic brick layers. (b) Detailed tension-induced fracture surface showing the pull-out of the lignocellulosic brick layers in the artificial wooden nacre (arrows). 


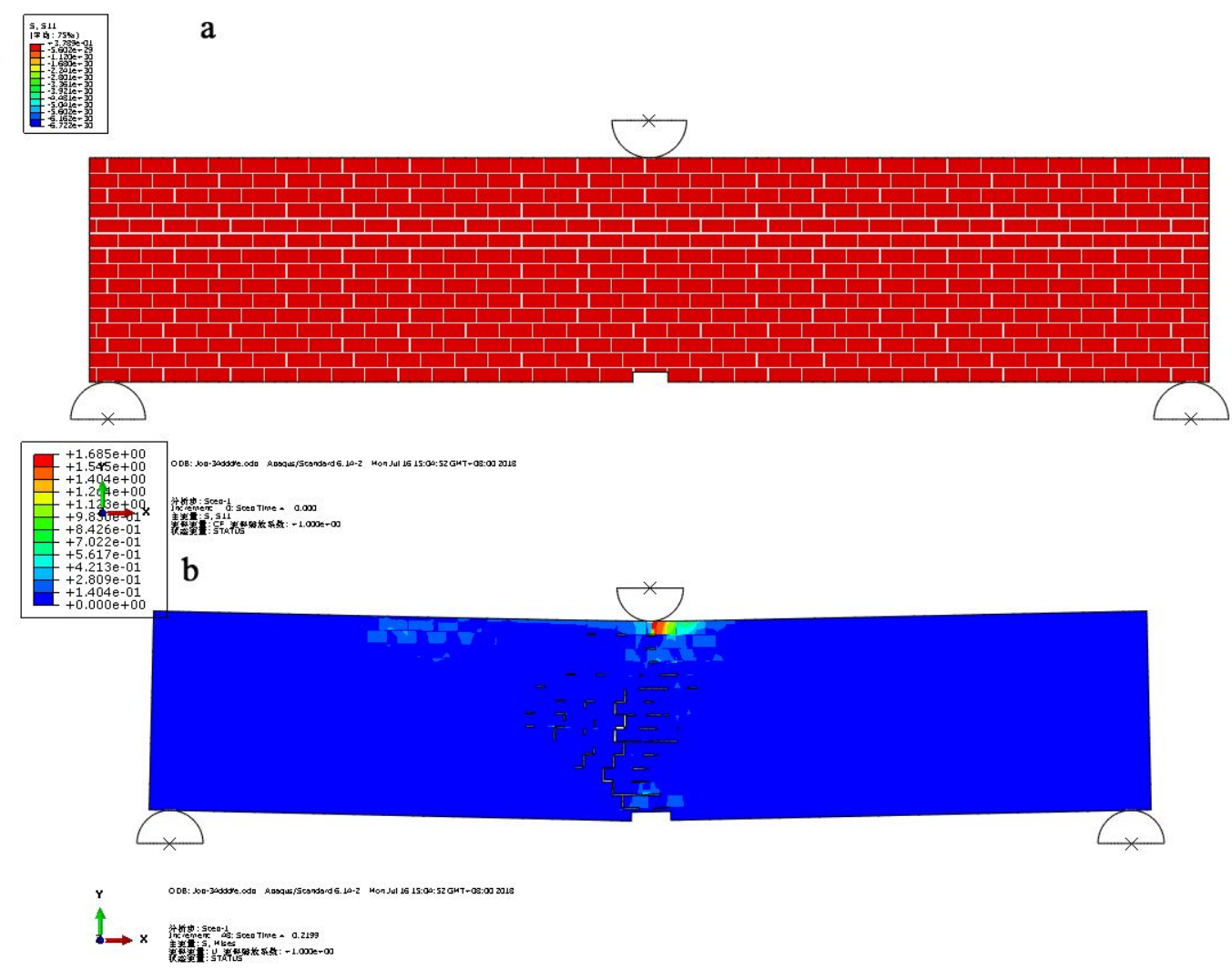

Figure S10. (a) The initial configuration of the 3D brick-and-mortar (BM) structure under three-point bending. (b) Simulated microcrack deflection and crack bridging in a typical 'brick-and-mortar' structure at a larger scale via nonlinear finite element model (FEM) simulation. 
a
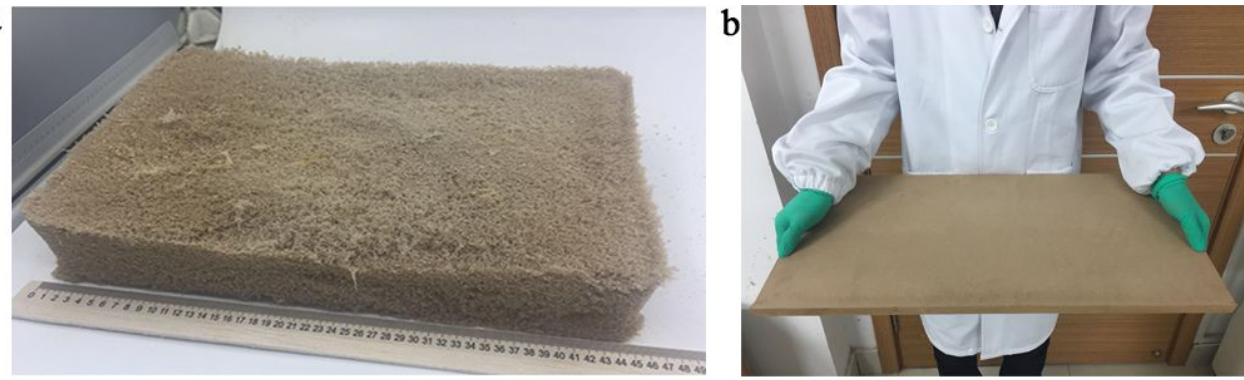

Figure S11. Photograph of large-sized (a) lignocellulosic building blocks matrix and (b) artificial wooden nacre. 
Table S1 Comparison of mechanical properties of artificial wooden nacre and their related materials.

\begin{tabular}{|c|c|c|c|}
\hline Materials & $\begin{array}{l}\text { Ultimate } \\
\text { flexural } \\
\text { strength } \\
(\mathrm{MPa})\end{array}$ & $\begin{array}{l}\text { Elastic } \\
\text { modulus } \\
\text { (GPa) }\end{array}$ & $\begin{array}{l}\text { Density } \\
\left(\mathrm{g} / \mathrm{cm}^{3}\right)\end{array}$ \\
\hline Blended mixture & $7.0 \pm 2.8$ & $4.2 \pm 1.8$ & $0.86 \pm 0.10$ \\
\hline $\begin{array}{c}\text { Pure densified } \\
\text { bulk }\end{array}$ & $17.8 \pm 3.4$ & $7.6 \pm 1.6$ & $0.82 \pm 0.07$ \\
\hline \multicolumn{4}{|l|}{ nanolignocellulose } \\
\hline $\begin{array}{l}\text { Disordered } \\
\text { composite }\end{array}$ & $32.1 \pm 3.9$ & $13.6 \pm 0.9$ & $0.98 \pm 0.12$ \\
\hline Without $\mathrm{CaCO}_{3}$ & $37.9 \pm 4.1$ & $18.8 \pm 1.8$ & $0.88 \pm 0.14$ \\
\hline Without PMMA & $46.0 \pm 4.8$ & $24.8 \pm 1.4$ & $1.14 \pm 0.09$ \\
\hline Pure PMMA & $55.9 \pm 5.5$ & $26.7 \pm 2.2$ & $1.23 \pm 0.12$ \\
\hline Natural AV nacre & $69.9 \pm 8.8$ & $38.5 \pm 2.5$ & $2.45 \pm 0.17$ \\
\hline Artificial wooden & $68.9 \pm 4.6$ & $25.5 \pm 1.8$ & $0.94 \pm 0.05$ \\
\hline nacre & & & \\
\hline
\end{tabular}


Table S2. Comparison of mechanical properties of artificial wooden nacre with other green plant fiber composites.

\begin{tabular}{|c|c|c|c|}
\hline Materials & $\begin{array}{l}\text { Strength } \\
(\mathrm{MPa})\end{array}$ & $\begin{array}{l}\text { Elastic } \\
\text { modulus } \\
\text { (GPa) }\end{array}$ & Reference \\
\hline Wheat straw (oxidation) & 25 & 2.1 & 1 \\
\hline Musacea bunch (steam exploded) & 24.1 & 0.2 & 2 \\
\hline Miscanthus sinensis/Kraft lignin & 61 & 0.8 & 3 \\
\hline Wood fibers (hydrothermal)/UF & 23.2 & 2 & 4 \\
\hline Wood fiber/Carbon fiber/PAM & 36.4 & 0.6 & 5 \\
\hline Wood fiber/ZnO/PE & 39.5 & 0.6 & 6 \\
\hline Wood fiber/ZnO/PP & 58.0 & 1.0 & 7 \\
\hline Bagasse fiber/UF & 18 & 2.5 & 8 \\
\hline Oil palm fiber/PF & 21.8 & 2.0 & 9 \\
\hline Wheat straw/UMF & 25 & 3.5 & 10 \\
\hline Artificial wooden nacre & $68.9 \pm 4.6$ & $25.5 \pm 1.8$ & Our work \\
\hline
\end{tabular}

\section{References}

1 Halvarsson, S.; Edlund, H.; Norgren, M. Properties of Medium-Density Fibreboard (MDF) Based on Wheat Straw and Melamine Modified Urea Formaldehyde (UMF) Resin. Ind. Crops Prod. 2008, 28, 37-46.

2 Quintana, G.; Velásquez, J.; Betancourt, S.; Gañán, P. Binderless Fiberboard from Steam Exploded Banana Bunch. Ind. Crops Prod. 2009, 29, 60-66. 
3 Velásquez, J. A.; Ferrando, F.; Salvadó, J. Effects of Kraft Lignin Addition in the Production of Binderless Fiberboard from Steam Exploded Miscanthus Sinensis. Ind. Crops Prod. 2003, 18, 17-23.

4 Ashori, A.; Nourbakhsh, A. Effects of Nanoclay as a Reinforcement Filler on the Physical and Mechanical Properties of Wood-Based Composite. J. Compos. Mater. 2009, 43, 1869-1875.

5 B. Dang, Y. Chen, N. Yang, B. Chen, Q. Sun. Effect of Carbon Fiber Addition on the Electromagnetic Shielding Properties of Carbon Fiber/Polyacrylamide/Wood Based Fiberboards. Nanotechnology 2018, 29, 195605.

6 Dang, B.; Chen, Y.; Shen, X.; Chen, B.; Sun, Q.; Jin, C. Fabrication of a Nano-ZnO/Polyethylene/Wood-Fiber Composite with Enhanced Microwave Absorption and Photocatalytic Activity via a Facile Hot-Press Method. Materials 2017, 10, 1267.

7 Ye, X.; Wang, H.; Zheng, K.; Wu, Z.; Zhou, H.; Tian, K.; Su, Z.; Tian, X. The Interface Designing and Reinforced Features of Wood Fiber/Polypropylene Composites: Wood Fiber Adopting Nano-Zinc-Oxide-Coating via Ion Assembly. Compos. Sci. Technol. 2016, 124, 1-9.

8 Lee, S.; Shupe, T. F.; Hse, C. Y. Mechanical and Physical Properties of Agro-Based Fiberboard. Holz Roh-Werkst. 2006, 64, 74-79.

9 Norul Izani, M. A.; Paridah, M. T.; Nor, M. M.; Anwar, U. Properties of Medium-Density Fibreboard (MDF) Made from Treated Empty Fruit Bunch of Oil Palm. J. Trop. For. Sci. 2013, 25, 175-183. 
10 Halvarsson, S.; Edlund, H.; Norgren, M. Properties of Medium-Density Fibreboard (MDF) Based on Wheat Straw and Melamine Modified Urea Formaldehyde (UMF) Resin. Ind. Crops Prod. 2008, 28, 37-46. 